\title{
Control of Aggregate Formation in Poly(3-hexylthiophene) by Solvent, Molecular Weight, and Synthetic Method
}

\section{Christina Scharsich, ${ }^{1}$ Ruth H. Lohwasser, ${ }^{2}$ Michael Sommer, ${ }^{2}$ Udom Asawapirom, ${ }^{3 *}$ Ullrich Scherf, ${ }^{3}$ Mukundan Thelakkat, ${ }^{2}$ Dieter Neher, ${ }^{4}$ Anna Köhler ${ }^{1}$}

${ }^{1}$ Organic Semiconductors, Experimental Physics II, Department of Physics and Bayreuth Institute of Macromolecular Science (BIMF), University of Bayreuth, Bayreuth 95440, Germany

${ }^{2}$ Applied Functional Polymers, Macromolecular Chemistry I, Department of Chemistry, University of Bayreuth, Bayreuth 95440, Germany

${ }^{3}$ Macromolecular Chemistry Group, Bergische Universität Wuppertal, Wuppertal 42097, Germany

${ }^{4}$ Soft Matter Physics, Institute of Physics and Astronomy, University of Potsdam, Potsdam 14476, Germany

Correspondence to: Anna Köhler (E-mail: anna.koehler@uni-bayreuth.de)

Received 26 July 2011; revised 7 November 2011; accepted 22 November 2011; published online 13 December 2011

DOI: $10.1002 /$ polb.23022

ABSTRACT: Aggregate formation in poly(3-hexylthiophene) depends on molecular weight, solvent, and synthetic method. The interplay of these parameters thus largely controls device performance. In order to obtain a quantitative understanding on how these factors control the resulting electronic properties of $\mathrm{P} 3 \mathrm{HT}$, we measured absorption in solution and in thin films as well as the resulting field effect mobility in transistors. By a detailed analysis of the absorption spectra, we deduce the fraction of aggregates formed, the excitonic coupling within the aggregates, and the conjugation length within the aggregates, all as a function of solvent quality for molecular weights from 5 to 19 $\mathrm{kDa}$. From this, we infer in which structure the aggregated chains pack. Although the $5 \mathrm{kDa}$ samples form straight chains, the 11 and $19 \mathrm{kDa}$ chains are kinked or folded, with conjugation lengths that increase as the solvent quality reduces. There is a maximum fraction of aggregated chains (about $55 \pm 5 \%$ ) that can be obtained, even for poor solvent quality. We show that inducing aggregation in solution leads to control of aggregate properties in thin films. As expected, the field-effect mobility correlates with the propensity to aggregation. Correspondingly, we find that a well-defined synthetic approach, tailored to give a narrow molecular weight distribution, is needed to obtain high field effect mobilities of up to $0.01 \mathrm{~cm}^{2} / \mathrm{Vs}$ for low molecular weight samples $(=11 \mathrm{kDa})$, while the influence of synthetic method is negligible for samples of higher molecular weight, if low molecular weight fractions are removed by extraction. (c) 2011 Wiley Periodicals, Inc. J Polym Sci Part B: Polym Phys 50: 442-453, 2012

KEYWORDS: conformational analysis; conjugated polymers; crystallization; films; interaction parameter; molecular weight distribution/molar mass distribution; nucleation; photophysics; structure; UV-vis spectroscopy
INTRODUCTION The optical and electronic properties of regioregular poly(3-hexylthiophene) (rrP3HT) are relevant for many applications in the field of organic semiconductors such as solar cells and field-effect transistors. Regioregular P3HT is known to show high mobilities that provide promising application on the industrial level. ${ }^{1-4}$ For this reason, the focus is often placed on the charge carrier mobility in P3HT thin films. It is well-known that several parameters affect the charge carrier mobility, such as the degree of crystallization, the molecular weight of P3HT chains, and the intermolecular coupling between these chains. ${ }^{4-7}$ Especially, the aggregation of polymer chains plays an important role in the performance of organic field effect transistors (OFETs) and organic solar cells. ${ }^{8}$
Indeed, the number and the nature of the aggregates are of central importance for these applications. Properties such as conjugation length, intermolecular coupling within the aggregates as well as the packing behavior of the polymer chains and disorder affect the applicable optic and electronic properties of P3HT. ${ }^{9-11}$ Another important parameter is the polydispersity of the polymer chains, which depends strongly on the synthetic approach. It was shown that low molecular weight polythiophene with low polydispersity forms highly ordered structures in thin films. ${ }^{12}$ Despite investigations on phase control in polymer films, the relation between molecular weight, polydispersity, and aggregation behavior of chains is still not fully understood. ${ }^{13}$

*Present address: National Nanotechnology Center (NSTDA), 130 Thailand Science Park, Phahonyothin Rd., Klong 1 Klong Luang, Pathumthani 12120, Thailand.

Additional Supporting Information may be found in the online version of this article.

(C) 2011 Wiley Periodicals, Inc. 
TABLE 1 Molecular Weights (MW) of Defined P3HTs Directly Synthesized to Get Well-Controlled Molecular Weights

\begin{tabular}{llllll}
\hline Poly(3-hexylthiophene) & $M_{\mathrm{n}}[\mathrm{Da}]$ & $M_{\mathrm{w}}[\mathrm{Da}]$ & PDI & $M_{\mathrm{n}}$ & DP \\
Based on & GPC & GPC & GPC & MALDI-TOF MS & MALDI-TOF MS \\
$5 \mathrm{kDa}$ & 5,100 & 6,300 & 1.22 & 3,200 & 19 \\
$11 \mathrm{kDa}$ & 11,300 & 15,200 & 1.11 & 7,100 & 43 \\
$19 \mathrm{kDa}$ & 18,600 & 21,600 & 1.16 & 12,400 & 74
\end{tabular}

$M_{\mathrm{n}}$, number-average molecular weight; $M_{\mathrm{w}}$, weight-average molecular Weight; PDI $=M_{\mathrm{w}} / M_{\mathrm{n}}$, polydispersity index; DP, degree of polymerization, i.e. the number of repeating units.

Here, we investigate the effect of molecular weight as well as the effect of synthetic approach to P3HT on the aggregate formation. We obtain information on the fraction of aggregates and on the packing behavior of P3HT chains within aggregates by combining spectroscopic methods with the theoretical works by Spano ${ }^{9}$ and Gierschner et al. ${ }^{11}$ Furthermore, we observe variations in excitonic coupling within P3HT aggregates and use this information to clarify to which extent the results obtained in solution can be transferred to P3HT thin films. We find that the synthetic approach affects the packing behavior of P3HT chains with low molecular weight. A synthesis defined to a specific molecular weight results in more accurate aggregates in both solution and thin films and thus in better FET performance. When going to higher molecular weights, these differences disappear, which implies that specific synthetic approaches to obtain defined high molecular weights, such as catalyst transfer polymerization, are not necessary. The early McCoullough route with subsequent extraction of low molecular weight fractions is sufficient to obtain comparable performances.

\section{EXPERIMENTAL}

The poly(3-hexylthiophene) samples differ in molecular weight and belong to two groups of different synthetic approach. The first group of poly(3-hexylthiophene)s was synthesized according to the catalyst transfer polymerization reaction method. ${ }^{14,15}$ These compounds, listed in Table 1, were tailor-made to their specific molecular weight. They have a very sharp molecular mass distribution and thus a very low polydispersity index (PDI). The number-average molecular weights $\left(M_{\mathrm{n}}\right)$ and the weight-average molecular weights $\left(M_{\mathrm{w}}\right)$ were measured by two methods. We used gel permeations chromatography (GPC) in tetrahydrofuran (THF) with polystyrene as calibration standard as well as matrix-assisted laser desorption ionization-time of flight mass spectroscopy (MALDI-TOF MS). Both methods are known to be associated with some experimental error. The molecular weight determined by MALDI-TOF MS is significantly lower than the one obtained from GPC measurements, because calibration against the polystyrene standard overestimates the molecular weight of rigid polymers. ${ }^{16}$ The degree of polymerization, DP, refers to the number of repeat units, and it is derived from the molecular weight as determined by MALDI-TOF, though we note that an uncertainty of about two repeat units remains. Throughout this article, the compounds in this group are referred to as "defined P3HT."
The second group of poly(3-hexylthiophene)s was synthesized according to the original McCullough route ${ }^{17}$ that does not allow for molecular weight control and that leads to broad molecular weight distributions. ${ }^{18}$ In order to obtain different molecular weight samples, the initial molecular mass distribution was fractionated with different solvents as listed in Table 2. Molecular mass distributions of the different samples were again obtained via GPC measurements with polystyrene standard. The compounds from this group are below referred to as "extracted P3HT." We consider the average degree of polymerization of each extracted compound to be similar to the DP of the corresponding defined compounds, because the $M_{\mathrm{n}}$ values of the defined and extracted samples are comparable in pairs.

Solutions were prepared from a ratio of good to moderately poor solvents, denoted subsequently as $A: B$, where $A$ gives the volume fraction of good solvent and $B$ the corresponding volume fraction of poor solvent, each as percent. Both solvents had a similar boiling point (bp) to eliminate different behaviors of volatilization. The good solvent for all compounds was chloroform $\left(\mathrm{CHCl}_{3}\right.$; bp $\left.=61{ }^{\circ} \mathrm{C}\right)$, whereas the moderately poor solvent was ethyl acetate (EtAc; bp $=77^{\circ} \mathrm{C}$ ). As both the boiling points are close enough, only the influence of solubility was studied. The worse solubility of P3HT in ethyl acetate results from its slightly higher polarity when compared with chloroform. The difference in Hildebrandt solubility parameter is not large $\left(9.10 \mathrm{cal}^{0.5} \mathrm{~cm}^{-1.5}\right.$ for EtAc versus $9.21 \mathrm{cal}^{0.5} \mathrm{~cm}^{-1.5}$ for $\mathrm{CHCl}_{3}$ ), yet it is sufficient to induce planarization (as evidenced further below by the absorption spectra). Importantly, the solubility of P3HT in the solvent mixtures at room temperature remains sufficiently high to ensure that the solution remains visibly clear, without any noticeable changes in the absorption spectra over a period of $24 \mathrm{~h}$. This is necessary to ensure the absorption spectra are not altered due to the effects of light scattering or changes of concentration due to a fall-out of

TABLE 2 Poly(3-hexylthiophene) Fractionated from a Broad Molecular Mass Distribution Sample with Different Solvents

$\begin{array}{lrrr}\text { Poly(3-hexylthiophene) Fractionated With } M_{\mathrm{n}}[\mathrm{Da}] & M_{\mathrm{w}}[\mathrm{Da}] & \text { PDI } \\ \text { Hexane } & 5,600 & 6,600 & 1.18 \\ \text { Dichloromethane } & 13,800 & 20,400 & 1.48 \\ \text { Chloroform } & 19,000 & 25,600 & 1.35\end{array}$

Molecular weights measured with GPC $\left(M_{\mathrm{n}}\right.$, number-average molecular weight; $M_{\mathrm{w}}$, weight-average molecular weight; PDI $=M_{\mathrm{w}} / M_{\mathrm{n}}$, polydispersity index). 
larger aggregates. Although the solutions do not change on the time scale of our experiments, they are not in thermal equilibrium, as detailed in the Supporting Information. This is in agreement with findings by $\mathrm{Xu}$ et al. on solvent-induced crystallization of related poly(thiophene)s using a mixture of chlorobenzene (solvent) and anisole (poor solvent). ${ }^{19}$

All solutions within one series had the same concentration. The defined compounds were studied at a concentration of $0.25 \mathrm{mg} / \mathrm{mol}$, whereas the solutions of the extracted P3HT samples had a concentration of $0.10 \mathrm{mg} / \mathrm{mol}$. For each molecular weight of the defined compounds, additional absorption measurements have also been carried out on a few samples at a concentration of $0.10 \mathrm{mg} / \mathrm{mol}$. This control experiment confirmed that the slight difference in concentration between the two series does not have any significant effect. In order to keep the concentrations within each series constant, every single solution within each solution series was prepared separately in new and isopropanol-rinsed dark sample glasses. Thereby, the P3HT was first completely dissolved in $\mathrm{CHCl}_{3}$ before the fraction of EtAc, the poor solvent, was added. The absorption of the solutions was measured in 1 mm-quartz cuvettes with a Varian Carry UV/Vis spectrometer in a standard double-beam transmission alignment. The absorption of thin films was measured with the same Varian Carry UV/Vis spectrometer by means of the diffuse reflectance accessory (integrating sphere). P3HT films were spin coated from solution with a concentration of $15 \mathrm{mg} / \mathrm{mL}$ at a constant speed of $1500 \mathrm{rpm}$ on Spectrosil B, a silica glass substrate. Prior to spin coating, the substrate was cleaned with common solvents such as acetone and isopropanol.

Organic field effect transistors were prepared in a bottom-gate, top-source/drain geometry under nitrogen atmosphere from solutions with a concentration of $10 \mathrm{mg} / \mathrm{mL}$. The substrates were n-doped silicon plates with a grown layer of insulating silicon dioxide of $\sim 300 \mathrm{~nm}$ thickness. Substrates were silanized using hexamethyldisilazane (HDMS) vapor for $1 \mathrm{~h}$ at $90{ }^{\circ} \mathrm{C}$ and $3 \mathrm{~h}$ at $60{ }^{\circ} \mathrm{C}$. Prior to silanization, the substrate surface was cleaned thoroughly with several common solvents and treated with oxygen plasma for $5 \mathrm{~min}$ at a power of $200 \mathrm{~W}$. Thereafter, the substrates were annealed under nitrogen atmosphere at $130{ }^{\circ} \mathrm{C}$ for $1 \mathrm{~h}$ and cooled down slowly to room temperature. After spin coating, 100-nm-thick gold electrodes were evaporated on top of the P3HT layer. The resulting field effect transistors had a channel width $W=14.85 \mathrm{~cm}$, a channel length $L=100 \mu \mathrm{m}$, and a capacitance of the insulating layer per unit area $C_{\mathrm{i}}=11.9 \mathrm{nF} / \mathrm{cm}^{2}$. The charge carrier mobilities were determined from a plot of the square root of the drain current in the saturation regime using

$$
I_{D S, s a t}=\frac{W C_{i}}{2 L} \mu_{s a t}\left(V_{G}-V_{T}\right)^{2}
$$

with $V_{\mathrm{T}}$ being the threshold voltage.

\section{RESULTS}

Figure 1 shows the absorption of rrP3HT in solutions of constant concentration. The solvent was a mixture of chloroform and ethyl acetate with increasing ratios of ethyl acetate. The latter is a moderately poor solvent for P3HT and is thus used to induce planarization/aggregation. Measurements were done for samples of three different molecular weights and made by two different synthetic approaches: rrP3HTs directly synthesized to get a specific molecular weight $(5,11$, and $19 \mathrm{kDa}$ ) and rrP3HT extracted from a large molecular mass distribution to get different molecular weight fractions (6, 14, and $19 \mathrm{kDa})$.

For both, defined and extracted P3HT samples, we observe two absorption features, an unstructured peak centered around $2.80 \mathrm{eV}$ and a band with structure at lower energy (three peaks at about 2.05, 2.20, and $2.40 \mathrm{eV}$ ). The lowenergy band increases in intensity with increasing fraction of poor solvent. Simultaneously, the ratio of the $0-0$ vibrational peak at $2.05 \mathrm{eV}$ to the $0-1$ vibrational peak at $2.20 \mathrm{eV}$ changes. With increasing molecular weight, the contribution of the low energy band to the overall spectrum increases. Furthermore, the high-energy band shifts slightly from 2.85 to $2.75 \mathrm{eV}$ when going from $5 / 6 \mathrm{kDa}$ to higher molecular weights. In contrast to the defined P3HT, the absorption of the high-energy band of the extracted P3HT with $6 \mathrm{kDa}$ shifts to lower energies $(2.80 \mathrm{eV})$ with increasing fraction of poor solvent before the low-energy band appears. The highenergy band has been identified as arising from coiled chains that form amorphous regions. The structured low-energy band is associated with absorption from planar chains that form weakly interacting H-aggregates. ${ }^{6,9}$

Although these spectra contain absorption due to both, aggregated and coiled chains, it is possible to extract the absorption spectra of the aggregated chains. As detailed in the Supporting Information, this is obtained by taking the spectra obtained in $100 \%$ chloroform solution (where the chains are fully dissolved and coiled), scaling it to the highenergy shoulder of the solution spectra (containing the absorption of aggregated and coiled chains), and subtracting it. We have also deduced the relative oscillator strength that describes the change in oscillator strength when going from a nonplanar chain as present in solution to a planar polymer chain in the aggregate, using the approach by Clark et al. ${ }^{20}$ This procedure is described further in the Supporting Information.

When the change in oscillator strength is known, the fraction of aggregated chains in the solution (as a total of the overall number of chains) can be derived from the fraction of absorption due to aggregated chains. This is shown in Figure 2. We observe that for the defined P3HT sample with $5 \mathrm{kDa}$ aggregation starts at $50 \%$ of poor solvent and increases linearly with decreasing solvent quality up to a fraction of $35 \%$ of aggregates. The difference to the extracted sample with comparable molecular weight is considerable. The latter does not aggregate below $70 \%$ of poor solvent and reaches only a fraction of $10 \%$ of aggregates at $90 \%$ of ethyl acetate. For the samples with 11 and $14 \mathrm{kDa}$, respectively, the aggregation starts much earlier. The defined sample shows aggregation at $15 \%$ of poor solvent and higher. Its fraction of 

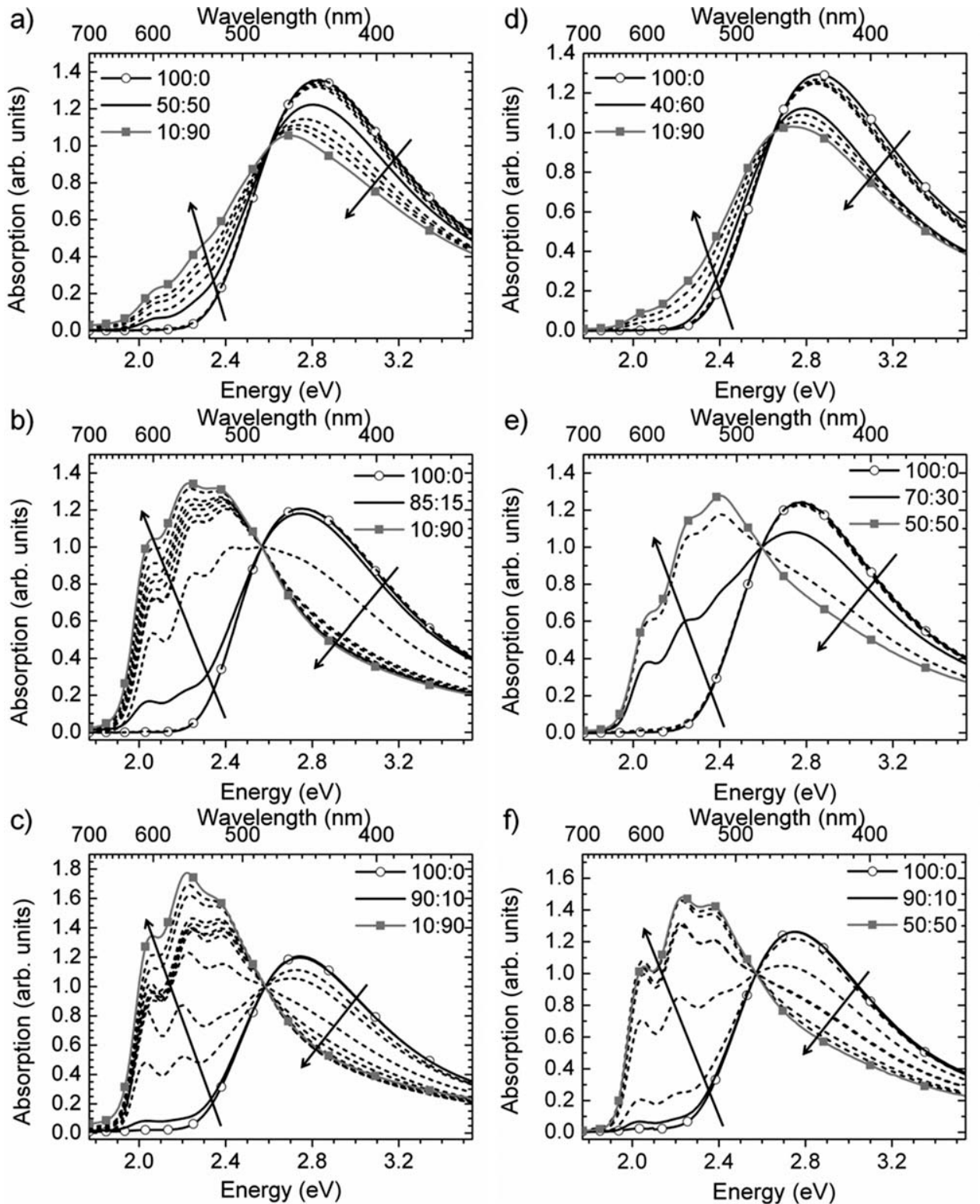

FIGURE 1 Absorption spectra of solutions with ratios of good:poor solvent $\left(\mathrm{CHCl}_{3}\right.$ :EtAc). Dashed lines show increments of about $10 \%$ of poor solvent. Solutions with maximum and minimum fraction of good solvent used are indicated as lines with open and solid symbols, respectively. The black solid line highlights the solvent fraction at which the onset of aggregation takes place. Adjacent graphs show samples of comparable molecular weight. Left column: Defined P3HTs with molecular weight (a) 5 kDa, (b) 11 kDa, (c) 19 kDa. Right column: Extracted P3HTs with molecular weight (d) 6 kDa, (e) 14 kDa, (f) 19 kDa.

aggregates increases steeply and reaches a saturation value of about 55\%, whereas the extracted sample with $14 \mathrm{kDa}$ starts to aggregate at $20 \%$ of poor solvent and shows fraction of aggregates below the defined sample. For the $19 \mathrm{kDa}$ samples from the defined and extracted synthetic approaches, aggregation starts at $10 \%$ of poor solvent and reaches as well a maximum value of about $50 \%$. No difference in aggregate fraction between the defined and extracted P3HT with $19 \mathrm{kDa}$ can be seen. The interesting feature to note is that for none of the samples the fraction of aggregated chains exceeds 55\%, even when $90 \%$ ethyl acetate is used. In other words, a substantial part of the chains remains dissolved. A $100 \%$ ethyl acetate solution becomes turbid, indicating the formation of larger agglomerated structures. The earlier onset of aggregation for P3HT with higher molecular weight, that is, longer chains, is consistent with Flory-Huggins theory that predicts less solubility for longer polymer chains. ${ }^{21}$

From Figure 2, it is evident that a substantial fraction of the chains forms aggregates in solution. It is known that such aggregates that are present in solution can later serve as nucleation points for aggregate formation in the film when prepared by spin-coating. ${ }^{13}$ To get a more thorough understanding on the nature of these aggregates, we now consider the spectral shape of the aggregate absorption. The aggregate absorption (derived as outlined in the Supporting 


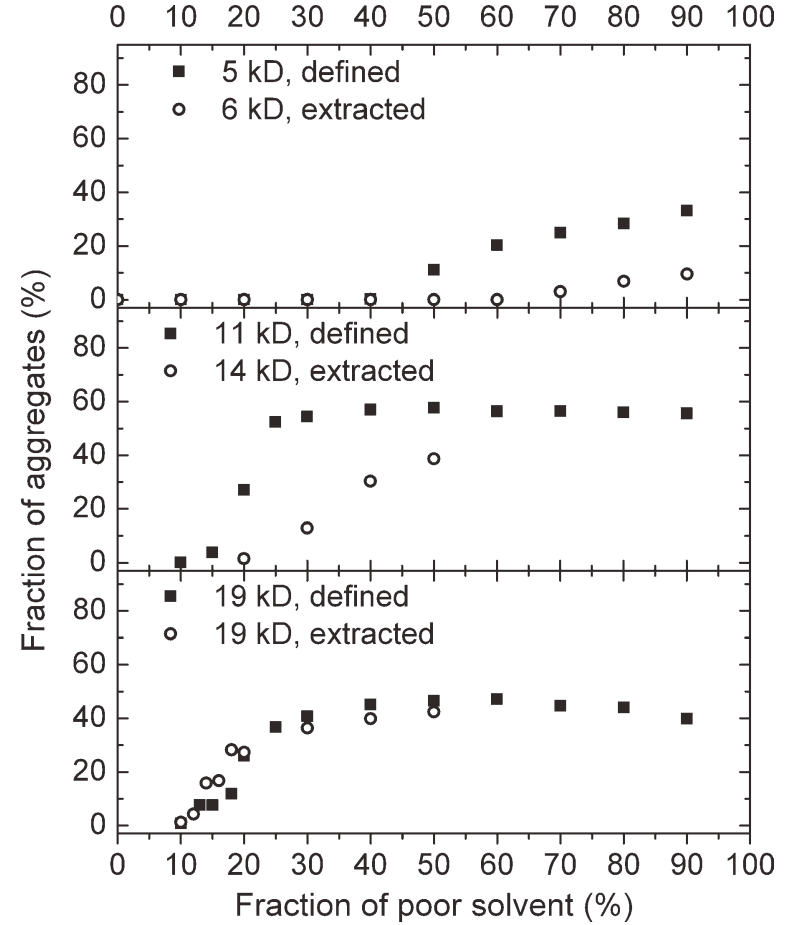

FIGURE 2 The fraction of aggregates present in solution as a function of poor solvent fraction for defined and extracted $\mathrm{P} 3 \mathrm{HT}$. The molecular weight increases from top to bottom.

Information), normalized to $0-1$ peak, is shown in Figure 3 for the defined P3HT samples. Very similar spectra can be obtained for the extracted samples. From Figure 3, we see, first, that the relative height of the 0-0 absorption peak increases with molecular weight. Second, the ratio of the $0-0$ to 0-1 absorption peak changes significantly with solvent quality. For the $5 \mathrm{kDa}$ sample the 0-0 intensity increases with increasing fraction of poor solvent. For the 11 and $19 \mathrm{kDa}$ samples, the 0-0 intensity first increases and then decreases with increasing fraction of ethyl acetate. The absorption spectra of the weakly interacting $\mathrm{H}$-aggregates can be analyzed in a framework for excitonic coupling in weakly interacting $\mathrm{H}$ aggregates that has been developed by Spano., ${ }^{9,22}$ According to this model, the relative height of the $0-0$ peak is a measure for the degree of excitonic coupling present in the aggregate. The very high 0-0 peak (0-0/0-1 ratios larger than unity) observed for the $19 \mathrm{kDa}$ sample at 90:10 EtAc: $\mathrm{CHCl}_{3}$ mixtures (line with open symbols in Fig. 3) thus implies excitonic coupling is largely absent, even though the chain must adopt conformations that are already partially planarized as evidenced by the low energy of the 0-0 peak. In contrast, excitonic coupling is particularly strong for the 50:50 mixture (black line) and then reduces somewhat when adding more ethyl acetate. Quantitatively, the 0-0/0-1 absorption peak ratio relates to the excitonic coupling $\mathrm{J}$ as follows

$$
\frac{A_{0-0}}{A_{0-1}}=\frac{n_{0-0}}{n_{0-1}} \frac{\left(1-\frac{J}{\omega_{0}} e^{-s} \sum_{v>0} \frac{S^{v}}{v ! v}\right)^{2}}{S\left(1-\frac{J}{\omega_{0}} e^{-s} \sum_{v \neq 1} \frac{S^{v}}{v !(v-1)}\right)^{2}},
$$

where $S$ is the Huang Rhys parameter of the single emitter and $\omega_{0}$ is its effective vibrational energy, $n_{0-0}$ and $n_{0-1}$ refer to the refractive index at the position of $A_{0-0}$ and $A_{0-1}$. To allow for an exact analysis, we have taken photoluminescence spectra and applied a Franck-Condon analysis in order to derive $S$ and $\omega_{0}$. Through this analysis, we found $S$ to be 0.90 (5 kDa sample) and 0.84 (11 and $19 \mathrm{kDa}$ samples) and $\omega_{0}$ to be $1390 \mathrm{~cm}^{-1}$. Full details of the Franck-Condon analysis are given as Supporting Information.

Using this approach, we obtained the excitonic couplings shown in Figure 4. We first consider the defined P3HT samples. For all compounds, we observe an initial rise of the excitonic coupling up to a peak followed by a decrease. For the $5 \mathrm{kDa}$ sample, the rise is not visible, only the decrease. For the extracted P3HT samples with 6 and $14 \mathrm{kDa}$, the excitonic coupling is slightly higher than for the comparable defined samples, whereas the defined and extracted samples with $19 \mathrm{kDa}$ show no significant difference in excitonic coupling. The excitonic coupling within the P3HT aggregates depends clearly on the fraction of the poor solvent and thus

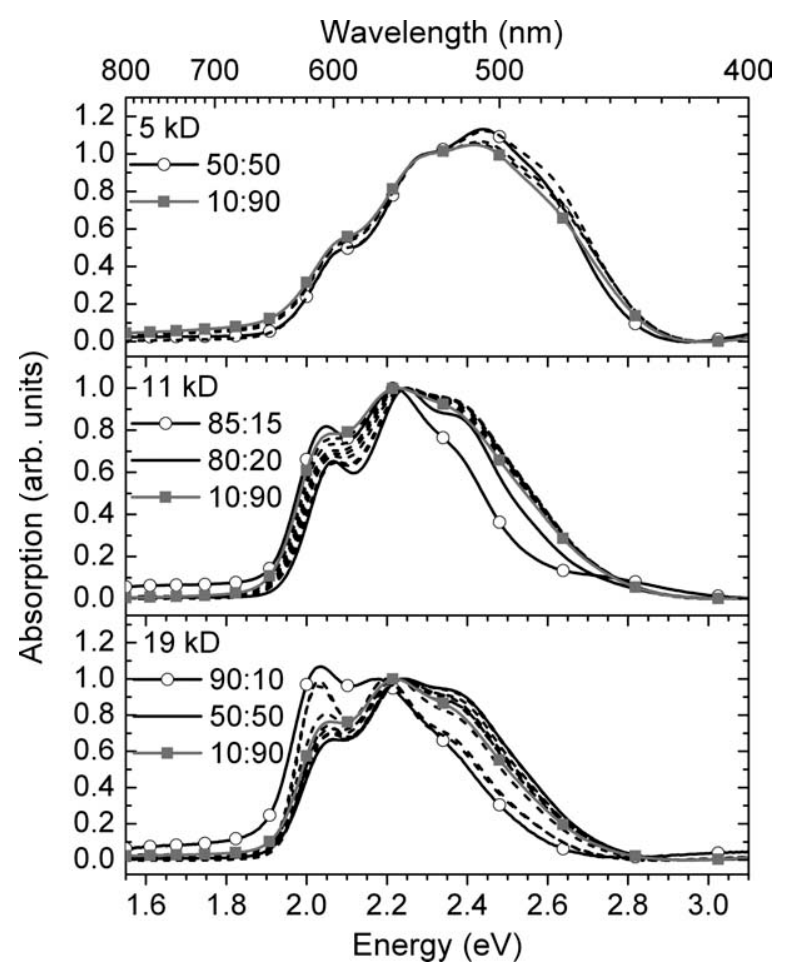

FIGURE 3 The absorption spectra of aggregates in solutions of defined P3HT. The spectra are normalized to the second absorption peak and presented for different fractions of poor solvent. The given ratios denote the volume fractions of good: poor solvent $\left(\mathrm{CHCl}_{3}\right.$ :EtAc). The dashed lines show increments of about $10 \%$ of poor solvent. Lines with open and solid symbols indicate samples with the minimum and maximum amount of poor solvent used. With increasing fraction of poor solvent, the relative intensity of the second absorption peak decreases up to the fraction indicated by the black line. From then onwards, it increases again. The molecular weight increases from top to bottom. 


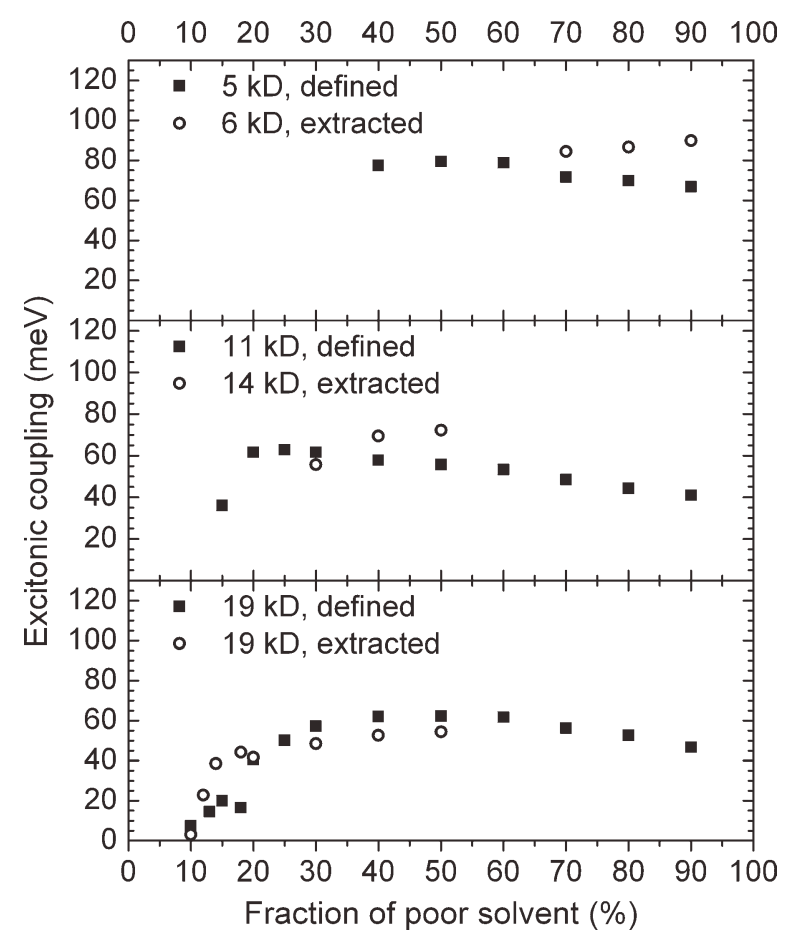

FIGURE 4 The excitonic coupling as function of poor solvent fraction for aggregates of defined and extracted P3HTs. The molecular weight increases from top to bottom. The excitonic coupling is calculated from the ratio of the first two absorption peaks according to Spano's model ${ }^{9}$ for weakly interacting $\mathrm{H}$ aggregates.

on solvent quality. We attribute this to a conjugation length dependence of the excitonic coupling. It is well-known that the strength of intermolecular coupling is related to the extent of the conjugated system. The breakdown of the point-dipole model for polymers is due to extended conjugations. Although for a point-dipole, the coupling between chains increases with conjugation length, this is not the case for very extended $\pi$-systems. Thus, a longer conjugation length implies a lower excitonic coupling and vice versa. ${ }^{10}$

By means of the theoretical work of Gierschner et al. who calculated the excitonic coupling in thiophene-based conjugated polymers as a function of repeating units, we derive the conjugation lengths within the aggregates of the defined and extracted P3HT as a function of poor solvent fraction. ${ }^{11}$ The excitonic coupling in regioregular P3HT is lower than the coupling in polythiophene without any side chains calculated by Gierschner et al. This leads to an overestimation of the conjugation length in rrP3HT, which was corrected by a factor of 0.8 . This factor takes into account that the conjugation length of the $5 \mathrm{kDa}$ sample cannot exceed the degree of polymerization of the polymer chains known from MALDITOF MS. Figure 5(a) shows the resulting conjugation length as a function of poor solvent for defined and extracted P3HT samples. In addition, the corresponding position of the $0-0$ absorption peak, taken from Figure 3, as well as the ratio of the first two absorption peaks is presented in Figure 5(b).
We find that the $5 / 6 \mathrm{kDa}$ samples have more or less constant conjugation lengths with increasing fraction of poor solvent, and that the extracted sample shows a shorter conjugation length than the defined P3HT sample. The defined samples with 11 and $19 \mathrm{kDa}$ show a minimum conjugation length at 25 and $50 \%$ of poor solvent, respectively, followed by a steady increase of conjugation length with decreasing solvent quality. Although the extracted sample with $19 \mathrm{kDa}$ shows a similar behavior to the $19 \mathrm{kDa}$ defined sample, the conjugation lengths of the $14 \mathrm{kDa}$ extracted sample has again a shorter conjugation length than the $11 \mathrm{kDa}$ defined sample. To assess the correlation to the degree of polymerization (DP), relative DP values were calculated from MALDITOF molecular weights. We like to point out that the $5 / 6$ $\mathrm{kDa}$ samples show conjugation lengths in the region of their degree of polymerization, whereas the samples with higher molecular weight form aggregates whose conjugation length is much lower than their degree of polymerization in the saturation region of the fraction of aggregates. Moreover, the positions of the $0-0$ absorption peaks correlate nicely with the conjugation lengths calculated from the excitonic coupling, given by the ratio of the $0-0 / 0-1$ absorption peak intensity, that is, the position of the first absorption peak shifts to maximum energy for solutions where the conjugation length calculated from the vibrational intensities is at a minimum. We note that these are two independently derived quantities.

From our investigations we know the fraction of aggregates (Fig. 2), their conjugation length (Fig. 5) and the degree of polymerization. Thus it is possible to infer information about the conformation of the aggregates. Our conclusions about the resulting conformations of the defined P3HT samples are summarized in Figure 6.

For the $5 \mathrm{kDa}$ sample, we consider the polymer chains within the aggregates to be completely planarized and conjugated. The aggregates coexist with still dissolved chains in the solution even when $90 \%$ of poor solvent is used. This picture is based on the fact that the conjugation length derived from the spectral analysis corresponds to the degree of polymerization obtained by the MALDI-TOF MS. Additionally, it is supported by a moderate fraction of aggregates which does not saturate for high amount of poor solvent. The still dissolved chains are likely to be the shorter ones in the overall distribution.

For the 11 and $19 \mathrm{kDa}$ samples, there are some key features to be noticed. In all cases the length of the conjugated segments is shorter than the total degree of polymerization [see Fig. 5(a)]. Thus, in principle, a polymer chain may comprise of one planarized segments and additional coiled segments or several planarized segments which are separated. This separation may be caused by coiled segments or simply by twists, kinks or folds.

Keeping this in mind, we now consider the $11 \mathrm{kDa}$ sample for $25 \%$ of poor solvent and more. At $25 \%$ of poor solvent, the conjugation length for each conjugated segment is about 22 repeating units which correspond to $50 \%$ of the degree 


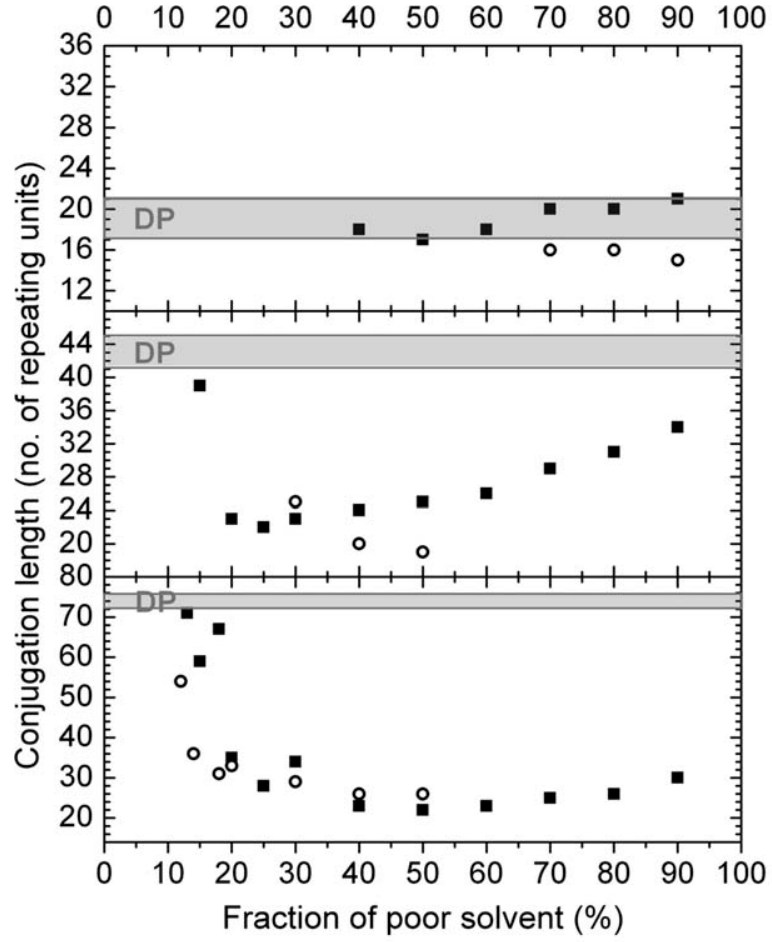

(a)

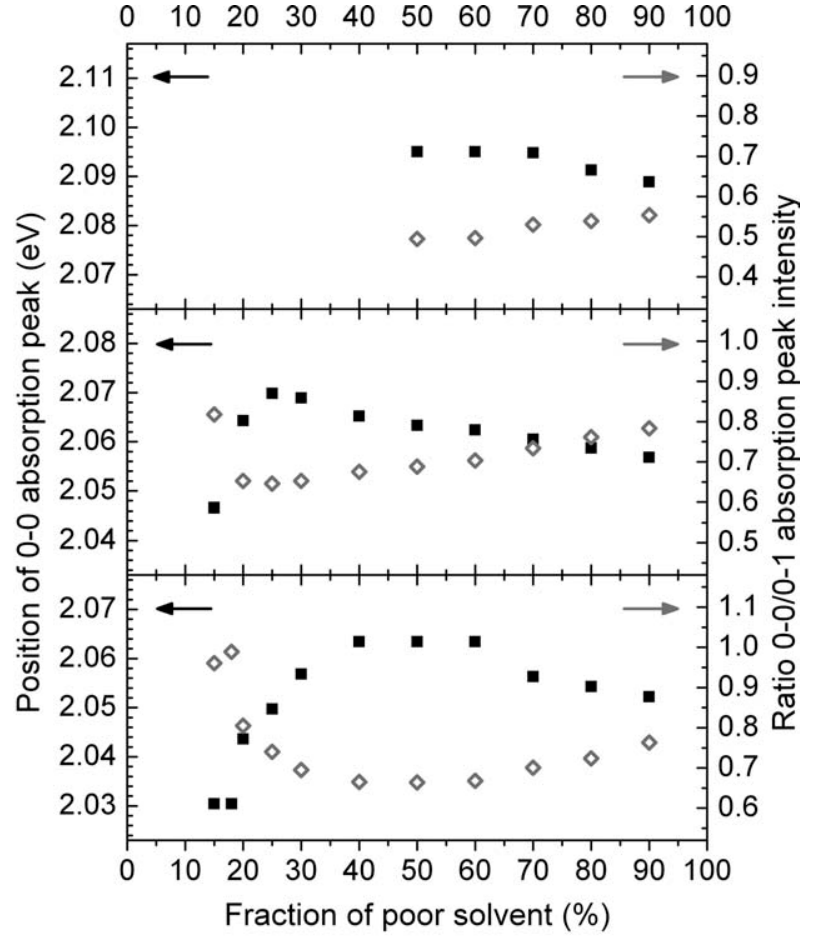

(b)

FIGURE 5 (a) The conjugation length derived from the excitonic coupling for chains of defined (solid squares) and extracted (open circles) $\mathrm{P} 3 \mathrm{HT}$, given as the number of thiophene repeating units. The grey bar indicates the degree of polymerization, determined from MALIDTOF with an error of \pm 2 repeat units, for reference. The molecular weight increases from top to bottom showing the pairs of 5/0.6 kDa, $11 /$ $14 \mathrm{kDa}$, and 19/19 kDa. (b) The position of the 0-0 absorption peak (solid symbols) and the ratio of the first to absorption peaks (open symbols) as a function of poor solvent fraction for the extracted P3HT samples with $M_{\mathrm{n}}=5 \mathrm{kDa}$ (top), $11 \mathrm{kDa}$ (center), and $19 \mathrm{kDa}$ (bottom).

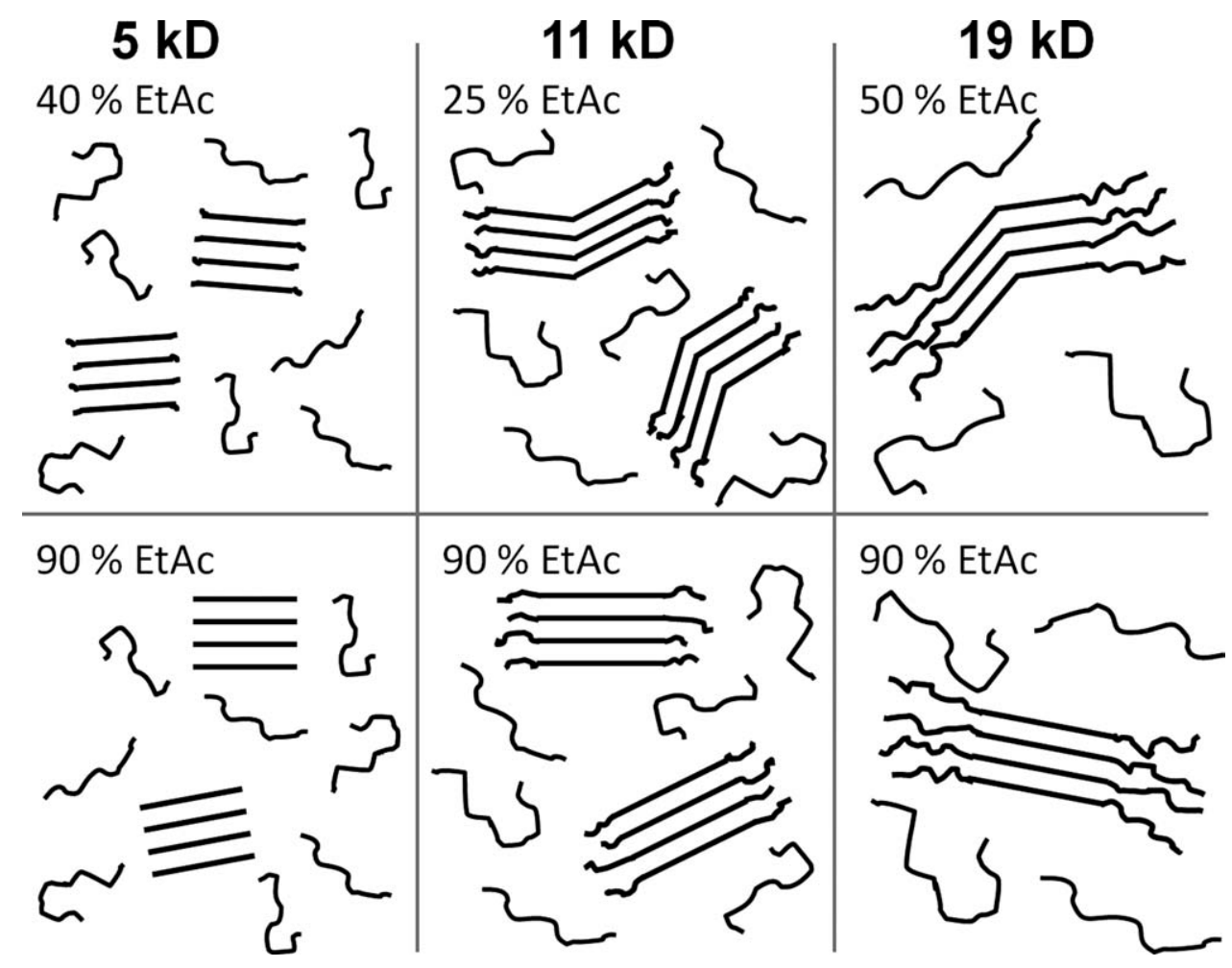

FIGURE 6 Possible scenarios for the packing behavior of defined P3HT chains for different molecular weights and different fractions of poor solvent. 
TABLE 3 Excitonic Coupling for Films of Defined and Extracted P3HT Spun from Solutions with Different Ratios of Good: Poor Solvent $\left(\mathrm{CHCl}_{3}\right.$ : EtAc)

\begin{tabular}{|c|c|c|c|}
\hline & $M_{\mathrm{n}}[\mathrm{Da}]$ & $\mathrm{CHCl}_{3}: \mathrm{EtAc}$ & $\begin{array}{l}\text { Excitonic } \\
\text { Coupling (meV) }\end{array}$ \\
\hline \multirow[t]{6}{*}{ Defined P3HT } & 5,100 & $100: 0$ & 70 \\
\hline & & $80: 20$ & 70 \\
\hline & 11,300 & $100: 0$ & 55 \\
\hline & & $85: 15$ & 45 \\
\hline & 18,600 & $100: 0$ & 35 \\
\hline & & $85: 15$ & 55 \\
\hline \multirow[t]{6}{*}{ Extracted P3HT } & 5,600 & $100: 0$ & 60 \\
\hline & & $80: 20$ & 70 \\
\hline & 13,800 & $100: 0$ & 40 \\
\hline & & $80: 20$ & 45 \\
\hline & 19,000 & $100: 0$ & 55 \\
\hline & & $80: 20$ & 35 \\
\hline
\end{tabular}

The excitonic couplings were determined by means of the model by Spano for weakly interacting $\mathrm{H}$-aggregates.

of polymerization. In principle, this still allows one or two conjugated segments in one polymer chain. When increasing the fraction of poor solvent, the conjugation length enlarges up to 34 repeating units being $80 \%$ of the DP. This implies that for higher amounts of poor solvents there is only one conjugated segment for each chain within the aggregates. On the other hand, the fraction of aggregates does not enlarge equally with the conjugation length but remains constant at $(55 \pm 5) \%$.

We now consider two scenarios.

1. If we assume that, at $25 \%$ of poor solvent, there is only one planarized segment per chain then $50 \%$ of each chain within the aggregates is still dissolved. Overall, the solution consists of $(55 \% \pm 5 \%)$ aggregated segments with the remainder being dissolved segments. In this scenario, the dissolved segments are then formed by half of each chain within the aggregates and some additional fully dissolved chains. Let us now consider a fraction of $90 \%$ of poor solvent in this scenario. At $90 \%$ of poor solvent, $80 \%$ of each chain within the aggregates is conjugated. Thus, only $20 \%$ of each chain within the aggregates can contribute to the fraction of dissolved segments in the solution. Compared to the situation with $25 \%$ of poor solvent, the overall fraction of aggregated segments in solution, and thus also of dissolved segments in solution, has not changed. As only $20 \%$ of each chain within the aggregates are dissolved, there must be more fully dissolved chains in the solution to make up the same total fraction of dissolved segments in solution than at $20 \%$ of poor solvent. An increase in the number of fully dissolved chains with increasing fraction of poor solvent, however, seems unlikely. Let us now turn to the second possible case.
2. Consider there are two planarized segments at $25 \%$ of poor solvent. By the same reasoning we find that much more fully dissolved chains are present at 25\% than at $90 \%$ of poor solvent. This seems more realistic.

Therefore, by comparing the degree of polymerization, the conjugation length and the fraction of aggregated segments present in solutions made with different fractions of poor solvent, we infer that the $11 \mathrm{kDa}$ sample adopts a conformation comprising predominantly two planarized segments at low fraction of poor solvent and one planarized segment at high fraction of poor solvent.

Very similar considerations are valid for the $19 \mathrm{kDa}$ sample, for which a conjugation length of 22 repeat units (as found for $50 \%$ ethyl acetate) allows for two or three planarized segments. For $90 \%$ ethyl acetate, where the conjugation length is 30 repeating units, there can be only one or two planarized segments.

In addition to studying P3HT solutions, we want to know whether the results obtained for P3HT aggregates in solution can be transferred to P3HT films that were spun from solution already containing aggregates due to poor solvent fractions. Therefore, we measured the absorption of the P3HT thin films (see Supporting Information). The same quantitative analysis of the ratio of the first two absorption peaks as for the absorption spectra of the P3HT solutions leads to the excitonic coupling within the aggregates according to the work of Spano. These excitonic couplings for the film aggregates are listed in Table 3 . We see that the aggregates of the defined P3HT in the film show very similar excitonic couplings as the aggregates found in solution for maximum aggregation (see Fig. 4). Therefore, we consider the aggregates in the defined P3HT films to be of the same nature as the aggregates in solution near maximum aggregation, or to be the same aggregates grown from aggregates in solution acting as nucleation points. Thus, we can control the

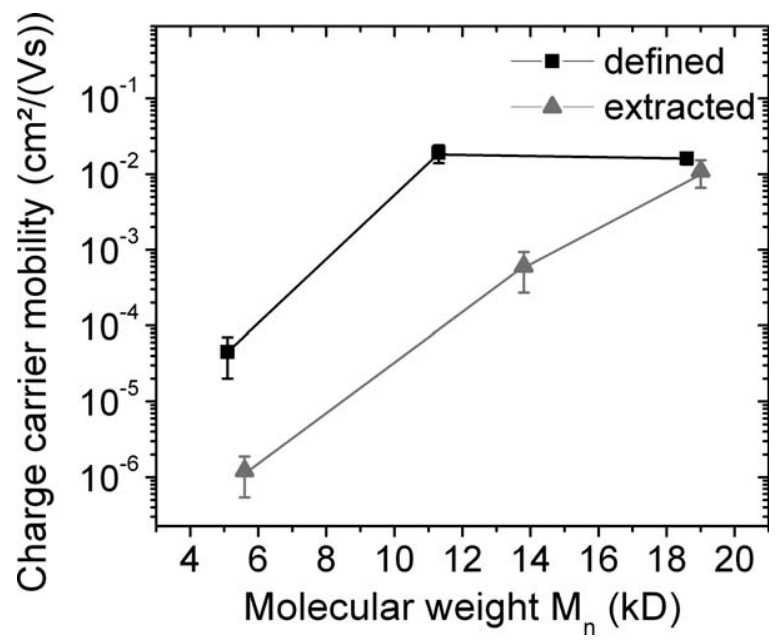

FIGURE 7 Charge carrier mobilities of defined and extracted P3HT samples as a function of molecular weight $M_{n}$. Films were spun from $100 \%$ chloroform solutions. 
formation of aggregates in the film via a control of solvent quality. This is true only for the defined P3HT samples.

As this control only applies for defined P3HT samples, we studied whether this advantage can be transferred also to charge carrier mobilities within the films. For this reason, we measured P3HT thin film transistors prepared from 100\% chloroform solutions simply by spin coating without any posttreatment of the devices such as annealing. The resulting saturation mobilities are shown in Figure 7 as a function of molecular weight. Both, the extracted and the defined samples, show increasing charge carrier mobilities with increasing molecular weight. This effect is well-known and was expected. Furthermore, the defined samples with lower molecular weight (5 and $11 \mathrm{kDa}$ ) reach significantly higher charge carrier mobilities than their comparable extracted samples. However, already for the samples with $19 \mathrm{kDa}$, this difference in charge carrier mobility vanishes. Both samples, defined and extracted, reach charge carrier mobilities of $10^{-2} \mathrm{~cm} / \mathrm{Vs}$.

\section{DISCUSSION}

\section{The Formation of P3HT Aggregates in Solution}

P3HT is well-known to exist in two distinct conformations, that is a random coil conformation leading to an amorphous film, and a planarized conformation giving rise to aggregates and films with corresponding crystalline inclusions. There have been various approaches to force poly(3-hexylthiophene) chains to aggregate. Several preparation parameters affect the solubility of polymer chains and thus the formation of aggregates, such as temperature, concentration or quality of the solvent used. ${ }^{23-25}$ Clark et al. used solutions with high concentrations of P3HT whose temperature dependent solubility lead to polymer aggregates at sufficiently low temperatures. ${ }^{6,20}$ A similar approach to induce aggregates in P3HT solutions was chosen by Liu et al. who investigated the crystallization behavior of P3HT in respect to crystallization temperature, polymer concentration and molecular weight of the samples. ${ }^{26}$ Likewise, Berson et al. and Oosterbaan et al. used highly concentrated and initially heated solutions of P3HT to induce P3HT nanofibers in the chilled solution and thus controlled the organization of the polymer chains. ${ }^{27,28}$ In contrast to that, Zhao et al. used ultrasonic oscillation to promote the formation of P3HT aggregates. ${ }^{29}$ Treatments such as thermal annealing, solvent vapor annealing, or the usage of high boiling point solvents enhance the order of the polymer chains within the aggregates. ${ }^{28}$ The approach by Park et al. refrained from any additional treatments. Park et al. ${ }^{30}$ made thin-film transistors from solutions containing small amounts of a nonsolvent with poly(3-hexylthiophene)s of molecular weights in the range of $M_{\mathrm{n}}=35-45 \mathrm{~kg} / \mathrm{mol}$.

In our studies, we aimed at inducing aggregates in P3HT of molecular weights in the range of $M_{\mathrm{n}}=5-19 \mathrm{~kg} / \mathrm{mol}$ in solution by varying the quality of the solvent. We studied the effect of different fractions of poor solvent, from 0 to $90 \%$, within the P3HT solutions with respect to molecular weight dependence and the influence of the synthetic approach. The induced aggregates in solution were analyzed carefully to obtain the fraction of polymer chains, which were aggregated due to low solvent quality. To relate the fraction of aggregate absorption to the actual fraction of aggregates, the relative oscillator strength was introduced that describes the relative increase in oscillator strength comparing a coiled P3HT chain with a planarized chain in an aggregate. This method was presented by Clark et al. in Ref. 31, where a relative oscillator strength of $1.39 \pm 0.10$ was obtained for solutions going from $70{ }^{\circ} \mathrm{C}$ to room temperature. In our investigations, we found a dependence of the relative oscillator strength on the fraction of poor solvent. To be precise, our investigations showed a minimum value for the relative oscillator strength in solutions with 30$50 \%$ of poor solvent. For higher amounts of poor solvent the oscillator strength increases slightly. This is direct evidence for an increase of conjugation length within the aggregates since oscillator strength is proportional to the squared transition dipole moment which increases linear with the dipole length. ${ }^{32}$

As for the fraction of aggregates, we showed that there is a limit to the maximum fraction of the polymer chains that can be forced to planarize and aggregate, even if $90 \%$ of poor solvent is used. This maximum fraction is about 50$60 \%$ for the 11 and $19 \mathrm{kDa}$ samples. This is in the order of the fraction of aggregated chains in solution induced by cooling published by Clark et al. being 39\% $\pm 10 \%{ }^{20}$ Obviously, there must be thermodynamic reasons that prevent the formation of entirely crystalline aggregates that would then precipitate from solution. One such factor may relate to the entropy of the system. For synthetic reasons, the connection between the first and the second 3-alkyl thiophenes unit is not head-to-tail, but instead it is head-to-head, inducing some disorder in each single chain.

As for the defined $5 \mathrm{kDa}$ sample, the onset of aggregation was lowered compared to the extracted $6 \mathrm{kDa}$ sample that did not show any aggregation below $70 \%$ of poor solvent. The defined $5 \mathrm{kDa}$ sample starts to aggregate already at $50 \%$ of poor solvent. Equally, the onset of aggregation is at higher fraction of poor solvent for the extracted $14 \mathrm{kDa}$ sample compared to the corresponding defined sample. Since the extracted sample was fractionized out of a very broad molecular mass distribution, the extracted sample consists of just those shorter chains that stopped growing during synthesis. It has been reported that this can result in lower regioregularity and thus in a relatively strong steric hindrance within the material. ${ }^{18}$ Moreover, the extracted samples usually have a broader polydispersity. The late onset of aggregation for extracted P3HT compounds with molecular weight below 19 $\mathrm{kDa}$ is thus comparable with the effect of a lower order on the packing of polymer chains. The higher the disorder, the lower is the crystallinity and the less ordered is the packing of the P3HT chains. ${ }^{33,34}$ No effect of synthetic approach on aggregation onset as well as on aggregate fraction can be seen for the $19 \mathrm{kDa}$ sample, because all chains with a smaller molecular weight are extracted out.

\section{The Conjugation-Length Dependence of Excitonic Coupling}

Considering the nature of the aggregates induced in solution, we showed that the excitonic coupling within the aggregates 
is a function of solvent quality. We interpret this dependency as a conjugation length-dependent dipole coupling as investigated theoretically by Gierschner et al. ${ }^{11}$ Indeed, the excitonic coupling of the aggregates in solution as a function of solvent quality show a maximum value for the defined 11 and $19 \mathrm{kDa}$ samples that correlates with a minimum in the conjugation length of the P3HT chains within the aggregates as evidenced through the minimum in the relative oscillator strength. In addition, the positions of the 0-0 absorption peaks in the isolated aggregate absorption spectra shift as a function of solvent quality, in agreement with the calculated conjugation lengths for these aggregates, giving direct experimental confirmation for the conjugation-length dependence of the excitonic coupling predicted theoretically (Fig. 5). This is indeed remarkable. For Förster-type energy transfer, the dependence of the dipole-coupling mechanism on the extent of the conjugated system is well documented through measurements of energy transfer rates. ${ }^{35,36}$ In contrast, experimental evidence for the manifestation of this effect in the case of excitonic coupling in aggregates is found only rarely.

In addition, we demonstrate that the excitonic coupling and accordingly the conjugation length depends on molecular weight and synthetic approach. We show that the defined synthetic approach results in higher conjugation lengths for P3HT compounds of molecular weights below $19 \mathrm{kDa}$ compared with extracted compounds, whereas for higher molecular weights, the synthetic approach is not relevant if low molecular weight fractions are extracted out. Although the effect of synthetic approach has not been studied yet, other effects, for example, the temperature dependence of the excitonic coupling in P3HT films were described in the literature. It is well known that temperature affects the conformation of P3HT chains. Pingel et al. showed that aggregates in P3dHT films have temperature dependent excitonic couplings. ${ }^{37}$ Furthermore, Clark et al. published a study on the effect of the boiling point of the solvent, which affects the rate with which the film dries after spinning from solution. ${ }^{20}$ Basically, the higher the solvent boiling point the lower is the resulting excitonic coupling and the higher is the obtained fraction of aggregates. This is in accordance with AFM results from Chang et al. who presented long P3HT ribbons in films spun from a high boiling point solvent. ${ }^{1}$ In contrast to these studies, we did not post-treat our samples to enhance the change in excitonic coupling and conjugation length. The resulting change in excitonic coupling in our study is merely due to thermodynamic effects that force the polymer chains to minimize their interaction with the surrounding solvent molecules and thus start aggregation with decreasing solvent quality. ${ }^{38}$

\section{The Packing Behavior of P3HT Aggregates in Solutions and Thin Films}

Combining the calculated conjugation length with the corresponding fraction of aggregates, we obtain a model for the packing behavior of the polymer chains in solution. We have found that the organization of P3HT chains depends not only on molecular weight but also on solvent quality. In particular, there is a solvent-dependent change in packing. Higher frac- tions of poor solvent lead to planarization of the aggregated middle sections of the polymer chains. For example, we conclude that the $5 \mathrm{kDa}$ sample packs as nonfolded chains. In contrast, the 11 and $19 \mathrm{kDa}$ sample form aggregates with kinks or even folded chains at lower fractions of poor solvent, while they form nonfolded chains at high fractions of poor solvent. This scenario is consistent with current understanding of the polymer crystallization process. ${ }^{25}$

This result further agrees with observations made by Liu et al. on the packing behavior of P3HT within nanofibers. ${ }^{26}$ By means of high-resolution transition electron microscopy, they obtained information on the width of nanoribbons. In combination with the contour length of the P3HT chains, this allows concluding on the packing behavior of the chains contained. Liu et al. showed for nanoribbons made of P3HT with $M_{\mathrm{n}}=9.3$ and $19.9 \mathrm{kDa}\left(M_{\mathrm{n}}\right.$-values corrected for GPC overestimation) that the low molecular weight sample packs indeed as nonfolded chains while chains in the high molecular weight sample have to be folded. ${ }^{26}$ This is also consistent with studies of $\mathrm{Wu}$ et al. on a series of P3HT with molecular weights of 5-19 $\mathrm{kDa}^{39}$

We also note that according to our results, the aggregates of the $5 \mathrm{kDa}$ compounds consist of completely planar chains implying that their conjugation length is equivalent to the full contour length of $7.5 \mathrm{~nm}$. A comparable low molecular weight sample with $M_{\mathrm{n}}=5.7 \mathrm{kDa}$ was recently studied by Brinkmann and Rannou. They reported on the packing behavior and the semicrystalline structures in P3HT and showed that the low molecular weight sample packs in crystalline lamellar of $7-8 \mathrm{~nm}$ width. ${ }^{40}$ This value confirms the conjugation length we obtained for the $5 \mathrm{kDa}$ sample. Thus, although our conclusions concerning the packing behavior of P3HT chains were based merely on spectroscopic studies, they are confirmed by the results on P3HT morphology from other groups.

For defined P3HT samples, we were able to transfer the aggregate interaction (excitonic coupling and conjugation length) obtained in solution into the thin film structure prepared by spin-coating. For example, in solution, the fraction of poor solvent controls the intermolecular coupling within the aggregates. For defined P3HT samples, we have found equal excitonic couplings in both, thin film aggregates and aggregates in solution. Thus, the aggregates induced in solution by changing the solvent quality are either the same aggregates found in the resulting film after spin-coating or they act at least as starting points for nucleation and growth. Therefore, a control over the excitonic coupling and conjugation length within aggregates in the resulting films is given by the control of the solvent quality.

Considering the charge carrier mobility in P3HT thin films, it is well known that the presence of aggregates or crystalline regions controls the mobility. Several aspects of this have been studied, among them the effect of crystallinity, ${ }^{39,41}$ the effect of the boiling point of the solvent, ${ }^{42,43}$ the effect of film thickness, ${ }^{44}$ the effect of the concentration of the solution $^{30}$ and the effect of the organization of the P3HT chains 
in the film. ${ }^{27}$ Our investigations concerning the charge carrier mobility in P3HT thin films focus on changes due to different molecular weights and different synthetic approaches. In addition to the well-known molecular weight dependence of charge carrier mobility, we observe a direct correlation between the fraction of aggregates in solution (Fig. 2) and the resulting thin film mobility (Fig. 7). For the defined and extracted $19 \mathrm{kDa}$ samples, the onset of aggregation in solution occurs early (at 10\% poor solvent), and in both cases similarly high fraction of aggregates up to $50 \%$ are obtained. The same is true for the defined $11 \mathrm{kDa}$ sample, where aggregates can take up even $60 \%$ in solution. For these three samples, the same charge carrier mobilities exceeding $10^{-2}$ $\mathrm{cm}^{2} / \mathrm{Vs}$ are obtained. For the extracted $14 \mathrm{kDa}$ sample, aggregation in solution takes place less easily, as evidenced by the higher fraction of poor solvent required and by the lower fractions of aggregates obtained. Concomitantly, the charge mobility is more than an order of magnitude lower than for the corresponding $11 \mathrm{kDa}$ defined sample. Aggregation is even more difficult for the $5 \mathrm{kDa}$ defined sample, translating equally in a reduced mobility until finally for the $6 \mathrm{kDa}$ extracted sample there is hardly any aggregation present in solution, and concomitantly the charge carrier mobility ranges at $10^{-6} \mathrm{~cm}^{2} /$ Vs. These changes in FET mobility cannot be attributed to differences in the excitonic coupling within the aggregates. Experimentally, comparison with Figure 4 shows that the changes in excitonic coupling with molecular weight and synthetic approach are less drastic. Furthermore, there is no a priori reason why a dipole-interaction based excitonic coupling should necessarily correlate with an exchange-interaction based charge transfer mechanism.

The observed correlation between the fraction of aggregates in solution and the thin film mobility may assist towards understanding the widely observed increase of charge carrier mobility with molecular weight. ${ }^{2,4}$ The reasons for the increase of mobility with molecular weight are still discussed. Zen et al. suggest that the increasing fraction of crystalline domains with increasing molecular weight controls the charge carrier mobility. ${ }^{41}$ In contrast, Brinkmann and Rannou suggest that an increasing fraction of bridging chains with increasing molecular weight increases the probability of charge transport through the less conductive amorphous domains of the film. ${ }^{45}$ Both approaches are based on the idea that the charge transport through the film is limited by amorphous domains. Recently, Lan and Huang published a theoretical study that implies that charge carrier mobilities below $10^{-2} \mathrm{~cm}^{2} / \mathrm{Vs}$ are controlled by the number of crossing points between the crystalline domains, whereas mobilities above this value correspond to present bridging chains between the ordered domains. ${ }^{46}$

The correlation we found between the fraction of aggregates in solution and the resulting thin film charge carrier mobility supports the arguments by Zen et al, at least for the range of molecular weights considered here. We observe the same high mobility for samples with very different molecular weight, that is, $11 \mathrm{kDa}$ and with $19 \mathrm{kDa}$, yet with the same propensity to aggregation. ${ }^{41}$ While the degree of aggregation in solution clearly does not need to translate into an equal aggregation in the film, it can serve as an indication of the propensity of the sample towards aggregation. The 11 and $19 \mathrm{kDa}$ chains have about 44 and 74 repeat units, respectively. When forming aggregates in solution at a medium fraction of poor solvent (i.e., a moderate driving force towards aggregation), both have a conjugation length of about 22 repeat units, implying one or at most two kinks in the chain. Thus, or a chain as short as $11 \mathrm{kDa}$, bridging chains between different crystallites are hard to imagine. Moreover, one would expect more bridging chains for the 19 $\mathrm{kDa}$ chain. As both samples show the same mobility this implies that either there are no bridging chains, or they do not contribute to the overall chain mobility. This argument is further strengthened by the fact that the mobility of $10^{-2}$ $\mathrm{cm}^{2} / \mathrm{Vs}$ we obtain for the defined $11 \mathrm{kDa}$ sample is the same mobility that is obtained by Kline et al. $^{2}$ for $40 \mathrm{kDa}$ samples, where bridging chains would be conceivable.

The authors thank Patrick Pingel and Rene Kalbitz for the FET measurements. Funding by the Graduiertenkolleg 1640 and SPP 1635 of the Deutsche Forschungsgemeinschaft is gratefully acknowledged. RHL thanks the Elite Netzwerk Bayern for a stipend according the Bayrisches Eliteförderungsgesetz.

\section{REFERENCES AND NOTES}

1 Chang, J. F.; Sun, B. Q.; Breiby, D. W.; Nielsen, M. M.; Solling, T. I.; Giles, M.; McCulloch, I.; Sirringhaus, H. Chem. Mater. 2004, 16, 4772-4776.

2 Kline, R. J.; McGehee, M. D.; Kadnikova, E. N.; Liu, J. S.; Frechet, J. M. J. Adv. Mater. 2003, 15, 1519-1522.

3 McCullough, R. D.; Tristramnagle, S.; Williams, S. P.; Lowe, R. D.; Jayaraman, M. J. Am. Chem. Soc. 1993, 115, 4910-4911.

4 Zen, A.; Pflaum, J.; Hirschmann, S.; Zhuang, W.; Jaiser, F.; Asawapirom, U.; Rabe, J. P.; Scherf, U.; Neher, D. Adv. Funct. Mater. 2004, 14, 757-764.

5 Chang, J. F.; Clark, J.; Zhao, N.; Sirringhaus, H.; Breiby, D. W.; Andreasen, J. W.; Nielsen, M. M.; Giles, M.; Heeney, M.; McCulloch, I. Phys. Rev. B 2006, 74, 115318.

6 Clark, J.; Silva, C.; Friend, R. H.; Spano, F. C. Phys. Rev. Lett. 2007, 98, 206406.

7 Reid, O. G.; Malik, J. A. N.; Latini, G.; Dayal, S.; Kopidakis, N.; Silva, C.; Stingelin, N.; Rumbles, G. J. Polym. Sci. Part B: Polym. Phys. 2012, 50, 27-37.

8 Herrmann, D.; Niesar, S.; Scharsich, C.; Köhler, A.; Stutzmann, M.; Riedle, E. J. Am. Chem. Soc. 2011, 133, 18220-18233.

9 Spano, F. C. J. Chem. Phys. 2005, 122, 234701.

10 Beljonne, D.; Cornil, J.; Silbey, R.; Millie, P.; Bredas, J. L. J. Chem. Phys. 2000, 112, 4749-4758.

11 Gierschner, J.; Huang, Y. S.; Van Averbeke, B.; Cornil, J.; Friend, R. H.; Beljonne, D. J. Chem. Phys. 2009, 130, 044105.

12 Pingel, P.; Zen, A.; Neher, D.; Lieberwirth, I.; Wegner, G.; Allard, S.; Scherf, U. Appl. Phys. A: Mater. Sci. Process. 2009, 95, 67-72.

13 Khan, A. L. T.; Banach, M. J.; Köhler, A. Synth. Met. 2003, 139, 905-907.

14 Sheina, E. E.; Liu, J. S.; lovu, M. C.; Laird, D. W.; McCullough, R. D. Macromolecules 2004, 37, 3526-3528. 
15 Lohwasser, R. H.; Thelakkat, M. Macromolecules 2011, 44, 3388-3397.

16 Conjugated Polymers: Theory, Synthesis, Properties, and Characterization; Skotheim, T. A.; Reynolds, J. R., Eds.; CRC Press, Taylor \& Francis Group: Boca Raton, Florida, 2007.

17 Loewe, R. S.; Khersonsky, S. M.; McCullough, R. D. Adv. Mater. 1999, 11, 250-253.

18 Trznadel, M.; Pron, A.; Zagorska, M.; Chrzaszcz, R.; Pielichowski, J. Macromolecules 1998, 31, 5051-5058.

19 Xu, W. T.; Li, L. G.; Tang, H. W.; Li, H.; Zhao, X. L.; Yang, X. N. J. Phys. Chem. B 2011, 115, 6412-6420.

20 Clark, J.; Chang, J. F.; Spano, F. C.; Friend, R. H.; Silva, C. Appl. Phys. Lett. 2009, 94, 163306.

21 Die Kunststoffe-Chemie, Physik, Technologie; Carlowitz B., Ed.; Carl Hanser Verlag: München, 1990.

22 Brown, P. J.; Thomas, D. S.; Kohler, A.; Wilson, J. S.; Kim, J. S.; Ramsdale, C. M.; Sirringhaus, H.; Friend, R. H. Phys. Rev. B 2003, 67, 064203.

23 Polymer Crystallization: Observations, Concepts and Interpretations; Reiter G., S. J. -U., Ed.; Springer: Berlin, 2003.

24 Progress in Understanding of Polymer Crystallization; Reiter G., S. G., Ed.; Springer: Berlin, 2007.

25 Hu, W. B.; Frenkel, D. In Interphases and Mesophases in Polymer Crystallization III; Springer-Verlag Berlin: Berlin, 2005. pp 1-35. 26 Liu, J. H.; Arif, M.; Zou, J. H.; Khondaker, S. I.; Zhai, L. Macromolecules 2009, 42, 9390-9393.

27 Berson, S.; De Bettignies, R.; Bailly, S.; Guillerez, S. Adv. Funct. Mater. 2007, 17, 1377-1384.

28 Oosterbaan, W. D.; Vrindts, V.; Berson, S.; Guillerez, S.; Douheret, O.; Ruttens, B.; D'Haen, J.; Adriaensens, P.; Manca, J.; Lutsen, L.; Vanderzande, D. J. Mater. Chem. 2009, 19, 5424-5435.

29 Zhao, K.; Xue, L. J.; Liu, J. G.; Gao, X.; Wu, S. P.; Han, Y. C.; Geng, Y. H. Langmuir 2010, 26, 471-477.

30 Park, Y. D.; Lee, H. S.; Choi, Y. J.; Kwak, D.; Cho, J. H.; Lee, S.; Cho, K. Adv. Funct. Mater. 2009, 19, 1200-1206.

31 Clark, J. EPAPS Document, EAPPLAB94058913 (= Supporting Information to Ref. 20).
32 Turro, N. J. Modern Molecular Photochemistry; University Science Books: Sausalito, California, 1991.

33 Kim, Y.; Cook, S.; Tuladhar, S. M.; Choulis, S. A.; Nelson, J.; Durrant, J. R.; Bradley, D. D. C.; Giles, M.; McCulloch, I.; Ha, C. S.; Ree, M. Nat. Mater. 2006, 5, 197-203.

34 Sirringhaus, H.; Brown, P. J.; Friend, R. H.; Nielsen, M. M.; Bechgaard, K.; Langeveld-Voss, B. M. W.; Spiering, A. J. H.; Janssen, R. A. J.; Meijer, E. W.; Herwig, P.; de Leeuw, D. M. Nature 1999, 401, 685-688.

35 Beljonne, D.; Curutchet, C.; Scholes, G. D.; Silbey, R. J. J. Phys. Chem. B 2009, 113, 6583-6599.

36 Beljonne, D.; Pourtois, G.; Silva, C.; Hennebicq, E.; Herz, L. M.; Friend, R. H.; Scholes, G. D.; Setayesh, S.; Mullen, K.; Bredas, J. L. Proc. Natl. Acad. Sci. USA 2002, 99, 10982-10987.

37 Pingel, P.; Zen, A.; Abellon, R. D.; Grozema, F. C.; Siebbeles, L. D. A.; Neher, D. Adv. Funct. Mater. 2010, 20, 2286-2295.

38 Hamley, I. W. Introduction to Soft Matter; Polymers, Colloids, Amphiphilies and Liquid Crystals; Wiley: Chichester, 2006.

39 Wu, Z. Y.; Petzold, A.; Henze, T.; Thurn-Albrecht, T.; Lohwasser, R. H.; Sommer, M.; Thelakkat, M. Macromolecules 2010, 43, 4646-4653.

40 Brinkmann, M.; Rannou, P. Macromolecules 2009, 42, 1125-1130.

41 Zen, A.; Saphiannikova, M.; Neher, D.; Grenzer, J.; Grigorian, S.; Pietsch, U.; Asawapirom, U.; Janietz, S.; Scherf, U.; Lieberwirth, I.; Wegner, G. Macromolecules 2006, 39, 2162-2171.

42 Cheng, H. L.; Lin, J. W.; Jang, M. F.; Wu, F. C.; Chou, W. Y.; Chang, M. H.; Chao, C. H. Macromolecules 2009, 42, 8251-8259.

43 Surin, M.; Leclere, P.; Lazzaroni, R.; Yuen, J. D.; Wang, G.; Moses, D.; Heeger, A. J.; Cho, S.; Lee, K. J. Appl. Phys. 2006, 100, 033712.

44 Joshi, S.; Grigorian, S.; Pietsch, U.; Pingel, P.; Zen, A.; Neher, D.; Scherf, U. Macromolecules 2008, 41, 6800-6808.

45 Brinkmann, M.; Rannou, P. Adv. Funct. Mater. 2007, 17, 101-108.

46 Lan, Y. K.; Huang, C. I. J. Phys. Chem. B 2009, 113, $14555-14564$. 